\title{
3 Research Square

\section{Hayabusa2 returned samples: A unique and pristine record of outer Solar System materials from asteroid Ryugu}

Motoo Ito ( $\sim$ motoo@jamstec.go.jp )

Japan Agency for Marine-Earth Science and Technology https://orcid.org/0000-0001-5686-0243

Naotaka Tomioka

Japan Agency for Marine-Earth Science and Technology https://orcid.org/0000-0001-5725-9513

Masayuki Uesugi

Japan Synchrotron Radiation Research Institute

Akira Yamaguchi

National Institute of Polar Research

Naoki Shirai

Tokyo Metropolitan University

Takuji Ohigashi

Institute for Molecular Science

Ming-Chang Liu

UCLA https://orcid.org/0000-0003-4030-5258

Richard Greenwood

The Open University

Makoto Kimura

National Institute of Polar Research

Naoya Imae

National Institute of Polar Research

Kentaro Uesugi

Japan Synchrotron Radiation Research Institute https://orcid.org/0000-0003-2579-513X

Aiko Nakato

Japan Aerospace Exploration Agency https://orcid.org/0000-0003-2392-1497

Kasumi Yogata

Institute of Space and Astronautical Science

Hayato Yuzawa

UVSOR/IMS https://orcid.org/0000-0003-4129-4407

\section{Yu Kodama}

Marine Works Japan, Ltd

Akira Tsuchiyama 
Ritsumeikan University

\section{Masahiro Yasutake}

Japan Synchrotron Radiation Research Institute

\section{Ross Findlay}

The Open University

\section{Ian Franchi}

The Open University https://orcid.org/0000-0003-4151-0480

\section{James Malley}

The Open University

\section{Kaitlyn McCain}

UCLA https://orcid.org/0000-0002-0811-135X

\section{Nozomi Matsuda}

UCLA

\section{Kevin McKeegan}

UCLA

\section{Kaori Hirahara}

Osaka University

\section{Akihisa Takeuchi}

Japan Synchrotron Radiation Research Institute

\section{Shun Sekimoto}

Kyoto University

\section{Ikuya Sakurai}

Nagoya University

\section{Ikuo Okada}

Nagoya University

\section{Yuzuru Karouji}

Japan Aerospace Exploration Agency https://orcid.org/0000-0002-8540-2986

\section{Masahiko Arakawa}

Kobe University https://orcid.org/0000-0003-3670-2699

\section{Atsushi Fujii}

Institute of Space and Astronautical Science https://orcid.org/0000-0003-0488-4969

\section{Masaki Fujimoto}

Japan Aerospace Exploration Agency

\section{Masahiko Hayakawa}

Japan Aerospace Exploration Agency

\section{Naoyuki Hirata}

Kobe University

\section{Naru Hirata}

The University of Aizu https://orcid.org/0000-0002-2435-604X 


\section{Rie Honda}

Kochi University

\section{Chikatoshi Honda}

The University of Aizu

\section{Satoshi Hosoda}

Institute of Space and Astronautical Science https://orcid.org/0000-0002-1964-2105

\section{Yu-ichi lijima}

deceased

\section{Hitoshi lkeda}

Japan Aerospace Exploration Agency

\section{Masateru Ishiguro}

Seoul National University https://orcid.org/0000-0002-7332-2479

\section{Yoshiaki Ishihara}

Japan Aerospace Exploration Agency https://orcid.org/0000-0002-0375-6300

\section{Takahiro Iwata}

Japan Aerospace Exploration Agency https://orcid.org/0000-0002-4613-7956

\section{Kosuke Kawahara}

Japan Aerospace Exploration Agency

\section{Shota Kikuchi}

Chiba Institute of Technology

\section{Kohei Kitazato}

University of Aizu https://orcid.org/0000-0002-4809-7492

\section{Koji Matsumoto}

National Astronomical Observatory of Japan

\section{Moe Matsuoka}

Japan Aerospace Exploration Agency

\section{Tatsuhiro Michikami}

Kindai University https://orcid.org/0000-0003-3096-5789

\section{Yuya Mimasu}

Institute of Space and Astronautical Science

\section{Akira Miura}

Japan Aerospace Exploration Agency https://orcid.org/0000-0002-9018-6077

\section{Osamu Mori}

Institute of Space and Astronautical Science https://orcid.org/0000-0001-9441-3356

\section{Tmokatsu Morota}

The University of Tokyo https://orcid.org/0000-0002-0500-4591

\section{Satoru Nakazawa}

Japan Aerospace Exploration Agency https://orcid.org/0000-0003-4250-1826

Noriyuki Namiki 
National Astronomical Observatory of Japan https://orcid.org/0000-0001-5374-372X

\section{Hirotomo Noda}

National Astronomical Observatory of Japan

\section{Rina Noguchi}

Niigata University

\section{Naoko Ogawa}

Japan Aerospace Exploration Agency https://orcid.org/0000-0002-7815-7791

\section{Kazunori Ogawa}

Kobe University https://orcid.org/0000-0002-4856-7108

\section{Tatsuaki Okada}

Japan Aerospace Exploration Agency https://orcid.org/0000-0001-6381-8107

\section{Chisato Okamoto}

deceased

\section{Go Ono}

Japan Aerospace Exploration Agency

\section{Masanobu Ozaki}

Japan Aerospace Exploration Agency https://orcid.org/0000-0002-1320-9781

\section{Takanao Saiki}

Japan Aerospace Exploration Agency

\section{Naoya Sakatani}

Rikkyo University https://orcid.org/0000-0003-4791-5227

\section{Hirotaka Sawada}

Japan Aerospace Exploration Agency

\section{Hiroki Senshu}

Chiba Institute of Technology

\section{Yuri Shimaki}

Japan Aerospace Exploration Agency https://orcid.org/0000-0001-8966-7650

\section{Kei Shirai}

Institute of Space and Astronautical Science

\section{Seiji Sugita}

The University of Tokyo https://orcid.org/0000-0001-6076-3614

\section{Yuto Takei}

Japan Aerospace Exploration Agency https://orcid.org/0000-0002-3900-1540

Hiroshi Takeuchi

JAXA https://orcid.org/0000-0001-5863-4653

\section{Satoshi Tanaka}

JAXA

\section{Eri Tatsumi}

Instituto de Astrofísica de Canarias https://orcid.org/0000-0002-6142-9842 


\section{Fuyuto Terui}

Kanagawa Institute of Technology.

\section{Ryudo Tsukizaki}

Japan Aerospace Exploration Agency https://orcid.org/0000-0002-6265-1672

\section{Koji Wada}

Chiba Institute of Technology https://orcid.org/0000-0002-6710-1768

\section{Manabu Yamada}

Chiba Institute of Technology https://orcid.org/0000-0003-0726-6592

\section{Tetsuya Yamada}

Japan Aerospace Exploration Agency

\section{Yukio Yamamoto}

Japan Aerospace Exploration Agency https://orcid.org/0000-0001-7172-1911

\section{Hajime Yano}

Institute of Space and Astronautical Science, Japan Aerospace Exploration Agency https://orcid.org/0000-0002-4125-0802

\section{Yasuhiro Yokota} Japan Aerospace Exploration Agency (JAXA) https://orcid.org/0000-0001-9230-974X

\section{Keisuke Yoshihara}

Japan Aerospace Exploration Agency

\section{Makoto Yoshikawa}

Japan Aerospace Exploration Agency

\section{Kent Yoshikawa}

Japan Aerospace Exploration Agency

\section{Ryota Fukai}

Japan Aerospace Exploration Agency https://orcid.org/0000-0002-1477-829X

\section{Shizuho Furuya}

University of Tokyo

\section{Kentaro Hatakeda}

Marine Works Japan, Ltd

\section{Tasuku Hayashi}

Japan Aerospace Exploration Agency

\section{Yuya Hitomi}

Marine Works Japan, Ltd.

\section{Kazuya Kumagai}

Marine Works Japan, Ltd

\section{Akiko Miyazaki}

Institute of Space and Astronautical Science https://orcid.org/0000-0001-7427-2285

\section{Masahiro Nishimura}

Institute of Space and Astronautical Science 
Hiromichi Soejima

Marine Works Japan, Ltd

\section{Ayako Iwamae}

Toyo University

\section{Daiki Yamamoto}

Japan Aerospace Exploration Agency

Miwa Yoshitake

Japan Aerospace Exploration Agency

Toru Yada

Japan Aerospace Exploration Agency https://orcid.org/0000-0002-7971-510X

\section{Masanao Abe}

Tomohiro Usui

Institute of Space and Astronautical Science https://orcid.org/0000-0002-4653-293X

\section{Sei-ichiro Watanabe}

Nagoya University https://orcid.org/0000-0002-5820-2102

\section{Yuichi Tsuda}

Japan Aerospace Exploration Agency

\section{Article}

\section{Keywords:}

Posted Date: January 28th, 2022

DOI: https://doi.org/10.21203/rs.3.rs-1270941/v1

License: (c) (1) This work is licensed under a Creative Commons Attribution 4.0 International License. Read Full License

Version of Record: A version of this preprint was published at Nature Astronomy on August 15th, 2022. See the published version at https://doi.org/10.1038/s41550-022-01745-5. 


\section{Abstract}

C-type asteroids likely formed in the outer Solar System and were then scattered inwards during giant planet migration (Walsh et al., 2011). They may have transported volatiles to the inner Solar System and created the conditions suitable for life on Earth(Alexander, 2017). Carbonaceous chondrites are fragments from C-type asteroids and provide evidence that these generally organic-rich (Garvie and Buseck, 2007) bodies experienced extensive aqueous alteration early in Solar System history (Alexander et al., 2014). On 6th December 2020, 5.4g of material was delivered to Earth from the C-type asteroid 162173 Ryugu by the Hayabusa2 spacecraft (Yada et al., 2021). Here we present the results of an integrated bulk and micro-analytical study of Ryugu particles, which provides a unique insight into the interrelationship between aliphatic-rich organics and surrounding hydrous minerals at a sub-micrometer scale. This dataset has clear implications for better understanding the origin and early evolution of Solar System organic matter and demonstrates that Ryugu particles are among the most uncontaminated extraterrestrial materials so far studied.

\section{Full Text}

Between June 2018 and November 2019, the Japan Aerospace Exploration Agency (JAXA) Hayabusa2 spacecraft undertook extensive remote sensing investigations of asteroid Ryugu. Data from the Near-IR Spectrometer (NIRS3) onboard Hayabusa2 indicated that Ryugu might be composed of material "similar to thermally and/or shock-metamorphosed carbonaceous chondrite meteorites" ${ }^{6}$; the closest match being the CY (Yamato-type) chondrites ${ }^{9}$. The low albedo of Ryugu can be explained by the presence of abundant carbon-rich components, in combination with grains size, porosity and space-weathering effects ${ }^{7}$.The Hayabusa2 spacecraft conducted two touchdowns and sample collections at Ryugu. One on $21^{\text {st }}$ of February obtained surface material that was stored in Chamber $A$ of the return capsule and a second, on $11^{\text {th }}$ of July of 2019 , collected material close to an artificial crater formed by a small carry-on impactor. These samples were stored in Chamber C. Initial, non-destructive characterization of the particles in a dedicated, contamination-free and pure nitrogen-filled chamber at the JAXA curation facility revealed that Ryugu particles were "most similar to $\mathrm{Cl}$ (Ivuna-type) chondrites" ${ }^{5}$ and displayed "a variety of alteration levels" ${ }^{\prime \prime}$. The seemingly contradictory classifications of Ryugu, as similar to either $\mathrm{CY}$ or $\mathrm{Cl}$ chondrites, can only be resolved by a detailed isotopic, elemental and mineralogical characterization of Ryugu particles. The results presented here provide a firm basis for deciding between these two preliminary interpretations of asteroid Ryugu's bulk composition.

Eight Ryugu particles ( $60 \mathrm{mg}$ in total), four from Chamber $\mathrm{A}$ and four from Chamber $\mathrm{C}$, were allocated to the Phase2 curation Kochi team. The principal goals of the research were to elucidate the nature, origin and evolutionary history of asteroid Ryugu, and to document the similarities and differences to other known extraterrestrial samples, such as chondritic meteorites, interplanetary dust particles (IDPs) and returned cometary samples collected by the NASA Stardust mission. 
Detailed mineralogical analysis of five Ryugu particles (A0029, A0037, C0009, C0014 and C0068), shows that they consist principally of fine- and coarse-grained phyllosilicates ( 64-88 vol\%, Figs. 1A,B and S1, Table S1). Anhydrous silicates (olivines, pyroxenes) that could be derived from chondrules and refractory inclusions are rare in the Ryugu particles examined in this study. Such anhydrous silicates have only been positively identified in particle C0009. Coarse-grained phyllosilicates (up to several tens $\mu \mathrm{m}$ in size) with feathery textures are embedded in the fine-grained phyllosilicate-rich matrix. The phyllosilicate grains are a serpentine-saponite intergrowth (Fig. 1C). A (Si+Al)-Mg-Fe plot further shows that bulk phyllosilicate matrices have intermediate compositions between serpentine and saponite (Figs. 2A,B). Carbonate minerals ( 2-21 vol\%), sulfide minerals ( 2.4-5.6 vol\%), and magnetite ( 3.6-6.8 vol\%) occur in the phyllosilicate matrix. Carbonates, as fragments in the matrix (< several hundred $\mu \mathrm{m})$, are mainly dolomites, with minor Ca-carbonate and breunnerite. Magnetites occur as isolated grains, framboids, plaquettes or spherical aggregates. Sulfides are mostly pyrrhotite showing irregular, hexagonal prism/plate or lath morphologies. Abundant submicron-sized pentlandites occur in the matrix or in association with pyrrhotite. Carbon-rich phases (<several to $10 \mu \mathrm{m}$ in size) occur ubiquitously in the phyllosilicate-rich matrix. Other accessory minerals are summarized in Extended Data Table 1. Mineral inventories identified by X-ray diffraction patterns of a mixture of A0029 and A0037, and C0087 are in good agreement with those identified in Cls (Orgueil meteorite), but completely different from the $\mathrm{CY}$ and CM (Mighei-type) chondrites (Extended Data Fig. 1 and Fig. S2). The bulk elemental abundances of Ryugu particles (A0098, C0068) are also consistent with those of $\mathrm{Cl}$ chondrites ${ }^{9}$ (Extended Data Fig. 2 and Table S1). In contrast, CM chondrites are characterized by a depletion in moderate- to highly-volatile elements, particularly $\mathrm{Mn}$ and $\mathrm{Zn}$, and higher abundances of refractory elements ${ }^{10}$. Some elements show highly variable concentrations, which may be a reflection of inherent sample heterogeneity due to the small size of the individual particles and consequential sampling biases. All petrological, mineralogical, and elemental characteristics indicate that the Ryugu particles are very similar to $\mathrm{Cl}$ chondrites ${ }^{11,12,13}$. The remarkable exception is the absence of ferrihydrite and sulfate in the Ryugu particles, which indicates that these minerals in $\mathrm{Cl}$ chondrites formed due to terrestrial weathering ${ }^{14}$.

The bulk oxygen isotopic composition of Ryugu particles A0098,2 and C0068,2 and a weighted average for the Hayabusa2 Chamber $C$ particles $(n=6)$ are shown in relation to Orgueil, Y-82162 (CY), CM, C2ungrouped meteorites ${ }^{15,16}$ in Figs. 2C,D. As is clear from this diagram the average composition of Chamber $\mathrm{C}$ particles plots close to that of Orgueil, but away from that of Y-82162 (Table S2). Compared to the average Chamber $C$ point, individual analyses for A0098,2 and C0068,2 have a lighter isotopic composition, which most likely reflects isotopic heterogeneity at the sampling scale involved. Both A0098,2 and C0068,2 were comparatively small particles. Compared to Orgueil, the $\Delta^{17} \mathrm{O}$ compositions of the individual Ryugu particles and the weighted Chamber $\mathrm{C}$ average analysis are somewhat higher, but there is overlap at the 2SD (standard deviation) level. In contrast, there is no overlap between the $\Delta^{17} \mathrm{O}$ value of Y-82162 and the Ryugu particles. These results further validate the potential link between Hayabusa2 samples and $\mathrm{Cl}$ chondrites, while appearing to exclude the possibility of a connection with the $\mathrm{CYs}$. The systematically higher $\Delta^{17} \mathrm{O}$ values of the Ryugu particles compared to Orgueil most likely reflects terrestrial contamination since the meteorites fall in 1864 . Weathering in the terrestrial 
environment ${ }^{11,14}$ would necessarily result in the incorporation of atmospheric oxygen and so pull the bulk analysis closer to the terrestrial fractionation line (TFL). This conclusion is in keeping with the mineralogical evidence discussed above that Ryugu particles do not contain ferrihydrite or sulfate, whereas Orgueil does.

Using coordinated microanalysis techniques (Fig. S3), we studied the spatial distribution of organic carbon throughout the entire surface area of the C0068-25 focused ion beam (FIB) section (Fig. 3A). Carbon-NEXAFS (near-edge X-ray absorption fine structure) spectra in the C0068-25 section show a variety of functional groups; aromatic or $\mathrm{C}=\mathrm{C}(285.2 \mathrm{eV}), \mathrm{C}=\mathrm{O}(286.5 \mathrm{eV})$, aliphatic $(287.5 \mathrm{eV})$, and $\mathrm{C}(=0) \mathrm{O}$ $(288.8 \mathrm{eV}$ ), without 1s-r^ exciton at $291.7 \mathrm{eV}$ of graphene structures (Fig. 3B), implying low degrees of thermal alteration. The aliphatic peak $(287.5 \mathrm{eV})$ of the organics in the C0068-25 is distinct from the previously studied insoluble organic matter (IOM) of carbonaceous chondrites ${ }^{17}$ and shows more similarities to IDPs ${ }^{18}$ and cometary particles obtained by the Stardust mission ${ }^{19}$. The aliphatic-rich organics areas are present locally within coarse-grained phyllosilicates, as well as areas with a poorly aromatic (or $\mathrm{C}=\mathrm{C}$ ) carbon structure (Figs. 3C,D). In contrast, A0002-23 and A0037-22, -23 sections display a lower abundance of aliphatic carbon-rich areas. The bulk mineralogy of these particles shows carbonate-rich lithologies similar to $\mathrm{Cls}^{20}$, indicative of more extensive parent body aqueous alteration (Extended Data Table 1). Oxidizing conditions would promote higher concentrations of carbonyl and carboxylic functional groups in the organic matter association with carbonates ${ }^{4}$. The sub-micrometerscale distribution of organics with an aliphatic carbon structure may vary significantly depending on the distribution of coarse-grained phyllosilicates. A hint of aliphatic bearing organics in association with phyllosilicate- $\mathrm{OH}$ has been reported in the Tagish Lake meteorite ${ }^{21}$. The coordinated microanalysis data suggest that aliphatic-rich organics may be widely distributed in C-type asteroids and exist in close association with phyllosilicates. This inference is consistent with the previous report of aliphatic/aromatic $\mathrm{CH}$ in the Ryugu particles demonstrated by the MicrOmega, a hyperspectral microscope operating in the near-infrared range ${ }^{8}$.

A kinetic study of organic matter degradation in the Murchison meteorite 22 may provide an important insight into the heterogeneous distribution of organics seen in the Ryugu particles. This study suggests that aliphatic $\mathrm{CH}$ bonds decrease with burial depth (maximum temperature of $\sim 30^{\circ} \mathrm{C}$ ) on the parent body. If the parent body did not reach $30^{\circ} \mathrm{C}$, then the initial distribution of aliphatic carbon-rich organics in phyllosilicates could be preserved. However, aqueous alteration on the parent body might complicate this scenario because carbonate-rich $\mathrm{A} 0037$ does not show any aliphatic carbon-rich regions associated with phyllosilicates. The $30^{\circ} \mathrm{C}$ temperature is broadly consistent with approximately $25^{\circ} \mathrm{C}$ value estimated from a comparison between chemical compositions of Fe-sulfides and phase equilibria in the Fe-Ni-S system $^{23}$.

A large nanoglobule was found in the C0068-25 section (n.g-1, Figs. 3A,B,C,E) showing highly aromatic (or $\mathrm{C}=\mathrm{C}$ ), moderately aliphatic, and weakly $\mathrm{C}(=0) \mathrm{O}$ and $\mathrm{C}=\mathrm{O}$ spectra. The aliphatic carbon feature does not match that of the bulk IOM and associated organic nanoglobules in chondritic meteorites (Fig. 
3B) ${ }^{4,24}$. Raman and infrared spectroscopies of nanoglobules in Tagish Lake show that they are composed of aliphatic and oxidized organic matter, and disordered polycyclic aromatic organic matter, as well more complex organic structures ${ }^{3,25}$. The aliphatic carbon feature indicated by the nanoglobule "n.g1 " may be an analytical artifact due to the surrounding matrix containing aliphatic-rich organics An important and as yet unresolved question is whether the unique nature of the aliphatic carbon-rich organics associated with coarse-grained phyllosilicates observed in this study is a feature found only in the Ryugu asteroid.

Nano-scale secondary ion mass spectrometry (NanoSIMS) ion images (Fig. 3F) of the C0068-25 section display relatively homogeneous variation in $\delta^{13} \mathrm{C}$ and $\delta^{15} \mathrm{~N}$, with the exception of a presolar graphite grain (P.G-1 in Fig. $3 \mathrm{~F}-\delta^{13} \mathrm{C}$ image) with extreme ${ }^{13} \mathrm{C}$ enrichment (Table S3). It is noteworthy that $\delta \mathrm{D}(841 \% \mathrm{o})$ and $\delta^{15} \mathrm{~N}(169 \%)$ values of aliphatic-rich organics associated with coarse-grained phyllosilicates are slightly higher than the average for the entire $C$ regions $\left(\delta D=528 \%\right.$, $\delta^{15} \mathrm{~N}=67 \%$ o) in C0068-25 (Table S3). This observation indicates that aliphatic-rich organics in coarse-grained phyllosilicates could be more primitive than the surrounding organics because of isotopic exchange with surrounding water in the parent body ${ }^{26}$. Aliphatic-rich organics might have formed from precursor molecules either in the protoplanetary disc or interstellar medium prior to Solar System formation via Fischer-Tropsh synthesis ${ }^{26}$, and were then slightly modified during aqueous alteration in the Ryugu (grand)parent body. Aliphatic-rich organics may have maintained their original isotopic ratios due to their association with coarse-grained phyllosilicates. However, the exact nature of the isotopically heavy carrier is still uncertain due to the close proximity of the various components. It could be either the aliphatic-rich organics or the surrounding coarse-grained phyllosilicates.

The hydrogen and nitrogen stable isotopic compositions of solar system objects demonstrate the existence of distinct cosmochemical reservoirs that were likely inherited from the solar nebula. These reservoirs correspond to the Sun, the inner Solar System, and the outer Solar System ${ }^{2,27}$. One possibility is that they were generated during the formation and subsequent migration of the giant planets, as envisaged in the Grand Tack hypothesis ${ }^{1}$. The potential role of giant planet migration within the early Solar System can be examined by determining whether asteroid Ryugu originated from outer Solar System materials, or whether it shares similarities with primitive meteorites and planets from the inner Solar System.

A bulk $\delta D$ and $\delta^{15} \mathrm{~N}$ plot of the A0002, A0037 and C0068 sections obtained by NanoSIMS is shown in Fig. 4 (Table S3) in comparison with other Solar System objects ${ }^{2,28,29}$. The Ryugu sections show no obvious correlation between $\delta^{13} \mathrm{C}$ and $\delta^{15} \mathrm{~N}$ (Table S3). Variations of bulk $\delta \mathrm{D}$ and $\delta^{15} \mathrm{~N}$ in the A0002, $\mathrm{A} 0037$ and C0068 sections are compatible with those seen in CR, C2-ung, IDPs and Wild2 cometary samples, but are higher than $\mathrm{CMs}$ and Cls (Fig. 4). The lower $\delta \mathrm{D}$ values for Cls compared to Ryugu particles may reflect the influence of terrestrial contaminations in the former ${ }^{30}$. The bulk $\delta D$ and $\delta^{15} \mathrm{~N}$ of the Ryugu sections tend to be lighter than the average values of Jupiter family and Oort cloud comets (Fig. 4), but with some exceptions due to aqueous alteration. Although the causes of the hydrogen and nitrogen isotopic 
heterogeneities observed in the Ryugu particles are not yet fully understood, due in part to the limited numbers of analyses so far available, the results from these isotopic systems still raise the possibility that Ryugu contains a significant portion of outer Solar System materials and shows some similarities to comets.

The delivery of volatiles (i.e., organics and water) to the Earth is still a subject of significant debate ${ }^{2}$. The aliphatic carbon-rich organics associated with coarse-grained phyllosilicates in Ryugu particles identified in this study likely represent one important source of volatiles. Organics incorporated into coarse-grained phyllosilicates seem to be more protected from degradation ${ }^{21,31}$ and breakdown ${ }^{32}$ than those in finegrained matrix during intensive heating events, such as meteoroid impacts on asteroid surfaces and terrestrial atmospheric entry. Because of the heavier hydrogen isotopic composition of the particles, they are unlikely to be the only source of volatiles to the early Earth. They may have been mixed with components having lighter hydrogen isotopic compositions, as recently proposed by the hypothesis of "solar-wind-derived water in silicates" 33 .

In this study we clearly demonstrate that (1): $\mathrm{Cl}$ meteorites, despite their geochemical importance as proxies of the bulk Solar System composition ${ }^{9,13}$, are biased and terrestrially-contaminated samples, and (2) we provide the first direct evidence of an interaction between aliphatic-rich organics and adjacent hydrous minerals. The findings of this study clearly demonstrate the importance of direct sampling of primitive asteroids and the need to transport returned samples in totally inert and sterile conditions. The evidence presented here shows that Ryugu particles are undoubtedly the most uncontaminated Solar System materials available for laboratory study and ongoing investigations of these precious samples will certainly expand our understanding of the early Solar System processes.

\section{References}

1. Walsh, K. J. et al. A low mass for Mars from Jupiter's early gas-driven migration. Nature $4 \mathbf{7 5}, \mathbf{2 0 6}$ 209 (2011).

2. Alexander, C. M. O'D. The origin of inner Solar System water. Phil. Trans. R. Soc. A 375, 20150384 (2017).

3. Garvie, L. A. J. \& Buseck, P. R. Prebiotic carbon in clays from Orgueil and Ivuna (Cl), and Tagish Lake (C2 ungrouped) meteorites, Meteorit. Planet. Sci. 42, 2111-2117 (2007).

4. Alexander, C. M. O. D. et al. Elemental, isotopic, and structural changes in Tagish Lake insoluble organic matter produced by parent body processes. Meteorit. Planet. Sci. 49, 503-525 (2014).

5. Yada, T. et al. Preliminary analysis of the Hyabusa2 samples returned from C-type asteroid Ryugu. Nat. Astron. https://doi.org/10.1038/s41550-021-01550-6 (2021).

6. Kitazato, K. et al. Surface composition of asteroid 162173 Ryugu as observed by the Hayabusa2 NIRS3 instrument. Science 364, 272-275 (2019). 
7. King, A. J. et al. The Yamato-type (CY) carbonaceous chondrite group: Analogues for the surface of asteroid Ryugu? Geochemistry, 79, 125531 (2019).

8. Pilorget, C. et al. First compositional analysis of Ryugu samples by the MicrOmega hyperspectral microscope. Nat. Astron. https://doi.org/10.1038/s41550-021-01549-z (2021).

9. Anders, E. \& Grevesse, N. Abundances of the elements: Meteoritic and solar. Geochim. Cosmochim. Acta 53, 197-214 (1989).

10. Wasson, J. T. \& Kallemeyn, G. W. Compositions of chondrites. Philos. Trans. R. Lond. 325, 535-544 (1988).

11. King, A. J. et al. Terrestrial modification of the Ivuna meteorite and a reassessment of the chemical composition of the $\mathrm{Cl}$ type specimen. Geochim. Cosmochim. Acta 268, 73-89 (2020).

12. Tomeoka, K. \& Buseck, P. R. Matrix mineralogy of the Orgueil $\mathrm{Cl}$ carbonaceous chondrite. Geochim. Cosmochim. Acta 52, 1627-1640 (1988).

13. Barrat, J.A. et al. Geochemistry of $\mathrm{Cl}$ chondrites: Major and trace elements, and $\mathrm{Cu}$ and $\mathrm{Zn}$ Isotopes. Geochim.Cosmochim. Acta 83, 79-92 (2012).

14. Gounelle, M. \& Zolensky, M. E. A terrestrial origin for sulfate veins in $\mathrm{Cl} 1$ chondrites. Meteorit. Planet. Sci. 36, 1321-1329 (2001).

15. Clayton, R. N. Oxygen Isotopes in Meteorites. Annu. Rev. Earth Planet. Sci. 21, 115-149 (1993).

16. Greenwood, R. C. et al. Melting and differentiation of early-formed asteroids: The perspective from high precision oxygen isotope studies. Chem. Erde-Geochem. 77, 1-43 (2017).

17. Cody, G. D. et al. Organic thermometry for chondritic parent bodies. Earth Planet. Sci. Lett. 272, 446455 (2006).

18. Keller, L. P. et al. The nature of molecular cloud material in interplanetary dust. Geochim. Cosmochim. Acta 68, 2577-2589 (2004).

19. Sandford, S. A. et al. Organics Captured from Comet 81P/Wild 2 by the Stardust Spacecraft. Science 314, 1720-1724 (2006).

20. Morlok A. et al. Brecciation and chemical heterogeneities of $\mathrm{Cl}$ chondrites. Geochim. Cosmochim. Acta 70, 5371-5394 (2006).

21. Yesiltas, M. \& Kebukawa, Y. Associations of organic matter with minerals in Tagish Lake meteorite via high spatial resolution synchrotron-based FTIR microspectroscopy. Meteorit. Planet. Sci. 51, 584-595 (2016).

22. Kebukawa, et al. Kinetics of organic matter degradation in the Murchison meteorite for the evaluation of parent-body temperature history. Meteorit. Planet. Sci. 45, 99-113 (2010).

23. Berger, E. L. et al. Heterogeneous histories of Ni-bearing pyrrhotite and pentlandite grains in the $\mathrm{Cl}$ chondrites Orgueil and Alais. Meteorit. Planet. Sci. 51, 1813-1829 (2016).

24. De Gregorio, B. T. et al. Isotopic and chemical variation of organic nanoglobules in primitive meteorites. Meteorit. Planet. Sci. 48, 904-928 (2013). 
25. De Gregorio B. T. et al. Isotopic anomalies in organic nanoglobules from comet 81P/Wild 2: Comparison to Murchison nanoglobules and isotopic anomalies induced in terrestrial organics by electron irradiation. Geochim. Cosmochim. Acta 74, 4454-4470 (2010).

26. Alexander, C. M. O. D. et al. The origin and evolution of chondrites recorded in the elemental and isotopic compositions of their macromolecular organic matter. Geochim. Cosmochim. Acta 71, 4380-4403 (2007).

27. Füri, E. \& Marty, B. Nitrogen isotope variations in the Solar System. Nature Geosci. 8, 515-522, https://doi.org/10.1038/ngeo2451 (2015).

28. Floss, C. et al. Identification of isotopically primitive interplanetary dust particles: A NanoSIMS isotopic imaging study. Geochim. Cosmochim. Acta 70, 2371-2399 (2006).

29. McKeegan K. D. et al. Isotopic compositions of cometary matter returned by Stardust. Science 314, 1724-1728 (2006).

30. Vacher, L. et al. Hydrogen in chondrites: Influence of parent body alteration and atmospheric contamination on primordial components. Geochim. Cosmochim. Acta 281, 53-66 (2020).

31. Salmon, V. et al. Protection of organic matter by mineral matrix in a Cenomanian black shale. Org. Geochem. 31, 463-474 (2000).

32. Tomioka, N. et al. Heating effects of the matrix of experimentally shocked Murchison CM chondrite: Comparison to micrometeorites. Meteorit. Planet. Sci. 42, 19-30 (2007).

33. Daly, L. et al. Solar wind contributions to Earth's oceans. Nat. Astron. 5, 1275-1285 (2021).

34. Zolensky, M. E. et al. Mineralogy and composition of matrix and chondrule rims in carbonaceous chondrites. Geochim. Cosmochim. Acta 57, 3123-3148 (1993).

35. Noguchi, T. et al. Variation of mineralogy and organic material during the early stages of aqueous activity recorded in Antarctic micrometeorites. Geochim. Cosmochim. Acta, 208, 119-144 (2017).

\section{Methods}

\section{Abbreviations}

EDS: energy dispersive $\mathrm{X}$-ray spectroscopy

EPMA: electron probe micro analysis

FFTC: facility-to-facility transfer container

FIB: focused ion beam processing

INAA: instrumental neutron activation analysis

NanoSIMS: nano-scale secondary ion mass spectrometry

SEM: scanning electron microscopy 
SR-XCT: synchrotron radiation-based computed tomography

SR-XRD-CT: synchrotron radiation-based X-ray diffractometry - computed tomography analysis

STXM-NEXAFS: scanning transmission X-ray microscope - near-edge X-ray absorption fine structure

TEM: transmission electron microscopy

XRD: X-ray diffraction

\section{Designs of analysis for Ryugu particles}

To obtain acquire complex micro-texture and chemical characteristics of the sample in sub-micrometer scale, we conducted coordinated micro analysis utilizing an SR-XCT \& XRD - FIB-XCT \& XRD - FIB STXM-NEXAFS - NanoSIMS - TEM analysis without degradation, contamination due to the terrestrial atmosphere and small particles, or mechanical sample damage. In parallel, we carried out systematic bulk analysis with SEM-EDS, EPMA, XRD, INAA, and a laser fluorination oxygen isotope facility. The analytical procedure is shown in Fig. S3, and each analysis was described in the following sections.

\section{Sample transportation and handling processes}

The Ryugu asteroid particles were recovered from the Hayabusa2 reentry capsule and transported to the JAXA Curation Facility at Sagamihara Japan without terrestrial atmospheric contamination (Yada et al., 2021). After initial and non-destructive characterizations at the JAXA Curation Facility, an airtight sample transport vessel (FFTC) and a sample capsule pack (made of sapphire glass and stainless steel with 10 $\mathrm{mm} \phi$ or $15 \mathrm{~mm} \phi$ depending on sample size) were used to avoid chemical reactions with the surrounding environment and/or terrestrial contaminants (e.g., water vapor, hydrocarbon, atmospheric gases, and small particles) and cross-contamination between samples during sample preparation and transportation among institutes and universities (Ito et al., 2020). To avoid degradation and contamination due to interaction with the terrestrial atmosphere (water vapor and oxygen gas), all the sample preparations including chipping by a tantalum chisel, cutting by a counter balanced diamond wire saw (Meiwa Fosis Corp. DWS 3400), and epoxy mount preparation) were conducted in a glove box in an atmosphere of pure, dry $\mathrm{N}_{2}$ (Dew point: -80 to $-60^{\circ} \mathrm{C}, \mathrm{O}_{2} \sim 50$ to $100 \mathrm{ppm}$ ). All items used here were cleaned by a combination of an ultra-pure water and ethanol under ultrasonication with different frequencies.

We studied meteorite collections (Orgueil, Yamato (Y)-791198, Y-82162, and Y 980115) of Antarctic meteorite center at the NIPR in this study.

For a transfer between instruments of SR-XCT, NanoSIMS, STXM-NEXAFS, and TEM, we used the universal holders for ultrathin film samples that described in previous studies (Ito et al., 2020; Uesugi et 
al., 2020; Shirai et al., 2020).

\section{A synchrotron radiation-based computed tomography analysis}

SR-CT analyses for Ryugu samples were performed with integrated computed tomography (CT) system at BL20XU/Spring-8. The integrated CT system consists of different measurement modes: wide-field and low-resolution $(\mathrm{WL})$ mode to capture the entire structure of the sample, narrow-field and high-resolution $(\mathrm{NH})$ mode for precise measurement of the region of interest, and X-ray diffraction (XRD) mode to acquire the diffraction pattern of bulk volume of the sample and perform XRD-CT to perform 2D mineral phase mapping of a horizontal plane in the sample. The X-ray detector for the WL mode (BM AA4OP; Hamamatsu Photonics) is equipped with a complementary metal oxide semiconductor (CMOS) camera, which has $4608 \times 4608$ pixels (C14120-20P; Hamamatsu Photonics), a scintillator consisting of a 10- $\mu$ mthick Lutetium Aluminum Garnet $\left(\mathrm{Lu}_{3} \mathrm{Al}_{5} \mathrm{O}_{12}: \mathrm{Ce}\right)$ foil, and relay lenses. The pixel size of the WL mode is approximately $0.848 \mu \mathrm{m}$. Thus, the field-of-view (FOV) of the WL mode is $\sim 6 \mathrm{~mm}$. The X-ray detector for the $\mathrm{NH}$ mode (BM AA50; Hamamatsu Photonics) is equipped with a scintillator consisting of Gadolinium Aluminum Gallium Garnet $\left(\mathrm{Gd}_{3} \mathrm{Al}_{2} \mathrm{Ga}_{3} \mathrm{O}_{12}\right)$ that is $200 \mu \mathrm{m}$ thick, the CMOS camera which has $2048 \times$ 2048 pixels (C11440-22CU; Hamamatsu Photonics), and a 20x lens. The pixel size of the NH mode is $\sim 0.25 \mu \mathrm{m}$ and the FOV is $\sim 0.5 \mathrm{~mm}$. The detector for XRD mode (BM AA60; Hamamatsu Photonics) is equipped with a scintillator consisting of a P43 $\left(\mathrm{Gd}_{2} \mathrm{O}_{2} \mathrm{~S}\right.$ : $\left.\mathrm{Tb}\right)$ powder screen that was $50 \mu \mathrm{m}$ thick, the CMOS camera which has $2304 \times 2304$ pixels (C15440-20UP; Hamamatsu Photonics), and relay lenses. Pixel size of the detector is $19.05 \mu \mathrm{m}$ and the FOV was $39 \mathrm{~mm}^{2}$. In order to increase the FOV, we applied the offset CT procedure in the WL mode. Transmitted light image for CT reconstruction was composed by combining images of 180 to 360 degrees which horizontally reflected around the rotation axis, with the images from 0 to 180 degrees.

For the XRD mode, an X-ray beam was focused by a Fresnel Zone Plate. The detector for the XRD mode was placed $110 \mathrm{~mm}$ behind the sample, with a $3 \mathrm{~mm}$ beam stop just in front of the detector. Diffraction images from $2 \theta=1.43^{\circ}$ to $18.00^{\circ}$ were obtained by the detector. Samples were vertically translated with a certain interval, and a half-rotated for each vertical scan step. Diffraction of mineral grains in a horizontal plane can be obtained if the mineral grains meet the Bragg condition in the half rotation. Diffraction images were then integrated into one image for each vertical scan step (Uesugi et al., 2020). The SR-XRDCT procedure is almost the same as for SR-XRD, except for the direction of scanning. The sample was scanned horizontally with half rotating of the sample. The SR-XRD-CT image was reconstructed using peak intensity of minerals as pixel value. Typically, samples were scanned with 500 to 1,000 steps for a horizontal scan.

The X-ray energy was fixed to $30 \mathrm{keV}$ for all experiments because it is the lower limit for X-ray penetration of meteorites that are $\sim 6 \mathrm{~mm}$ in diameter (Uesugi et al., 2010; Uesugi et al., 2013). The number of images acquired for all CT measurements during the half rotation was 1800 (3600 for offset CT 
procedure), and the exposure time for an image was $100 \mathrm{~ms}$ for the WL mode, $300 \mathrm{~ms}$ for the NH mode, $500 \mathrm{~ms}$ for XRD, and $50 \mathrm{~ms}$ for XRD-CT. The typical scanning time for one sample in WL mode was 10 min, in NH mode was 15 min, 3 hours for XRD, and 8 hours for SR-XRD-CT.

CT images were reconstructed by convolution-backprojection (CBP) method and normalized for 0 to 80 $\mathrm{cm}^{-1}$ of linear attenuation coefficient. Slice software was applied for the analysis of 3D data, and muXRD software was used for the analysis of XRD data.

\section{Optical, SEM-EDS and EPMA analyses}

The epoxy mount Ryugu particles (A0029, A0037, C0009, C0014, and C0068) were polished on a surface in a stepwise manner down to the level of a $0.5 \mu \mathrm{m}$ diamond lapping film ( $3 \mathrm{M}$ company) under dry condition avoiding elution of any materials from a surface during polishing. A polished surface of each sample was examined first by an optical microscope, and then by a JEOL JSM-7100F scanning electron microscope (SEM) equipped with an energy dispersive spectrometer (EDS) (AZtec energy) to obtain an overview of the mineralogy and textures of the samples by high-resolution imaging and qualitative chemical mapping at NIPR. Major and minor elemental abundance analysis of each sample were conducted with an electron probe microanalyzer (EPMA, JEOL JXA-8200). A series of natural and synthetic mineral standards were used for external calibration of EPMA performed at typical operating conditions of accelerating voltage of $15 \mathrm{kV}$ and beam current of $5 \mathrm{nA}$ (carbonates, phosphates, olivine, pyroxene, magnetite, and sulfide) or $30 \mathrm{nA}$ (natural and synthetic standards).

\section{High precision oxygen isotopic analysis}

Oxygen isotopic analysis was undertaken at the Open University (Milton Keynes, UK) using an infrared laser-assisted fluorination system (Miller et al., 1999; Greenwood et al., 2017). Four distinct Hayabusa2 samples were transported to the Open University in two sealed, nitrogen-filled "FTTC: facility-to-facility transport container" (Ito et al., 2020). One of the two FFTC contained grains from the initial Hayabusa2 touchdown collection (particle A0098,2: 5 grains), the other FFTC contained three sets of particles from the second, post impactor collection: C0014,2 1 particle $5.5 \mathrm{mg}$; C0068,2 1 particle $0.5 \mathrm{mg} ; \mathrm{C} 0087,2$ approximately 10 grains, $0.8 \mathrm{mg}$. Both holders were stored at the Open University in a dedicated cabinet with a continuously purged nitrogen atmosphere. The cabinet was housed within a secure class 100 cleanroom.

Sample loading was undertaken in a nitrogen "glove box" with monitored oxygen levels below $0.1 \%$. A new Ni sample holder was fabricated for the Hayabusa2 analysis work that consisted of just two sample wells ( $2.5 \mathrm{~mm}$ diameter, $5 \mathrm{~mm}$ depth), one for the Hayabusa2 particle and the other for the internal obsidian standard. During analysis, the sample well containing the Hayabusa2 material was overlain by a 
$\sim 1 \mathrm{~mm}$ thick, $3 \mathrm{~mm}$ diameter internal BaF2 window to retain the sample during laser reaction. The flow of BrF5 to the sample was maintained by gas mixing channels scribed into the Ni sample holder. The sample chamber configuration was also modified so that it could be removed from the fluorination line under vacuum and then opened within the nitrogen-filled glove box. The two-part chamber is made vacuum tight using a compression seal with a copper gasket and quick-release KFX clamp (Miller et al., 1999; Greenwood et al., 2017). A $3 \mathrm{~mm}$ thick $\mathrm{BaF}_{2}$ window at the top of the chamber allows simultaneous viewing and laser heating of samples. Following sample loading the chamber was then reclamped and reattached to the fluorination line. Prior to analysis the sample chamber was heated overnight under vacuum to a temperature of about $95^{\circ} \mathrm{C}$ to remove any adsorbed moisture. Following overnight heating, the chamber was allowed to cool to room temperature and then the section that had been brought up to atmosphere during the sample transfer process was purged using three aliquots of $\mathrm{BrF}_{5}$ to remove any moisture. These procedures ensured that the Hayabusa2 samples were never opened to the atmosphere or contaminated with moisture from those parts of the fluorination line that had been brought up to atmosphere during the sample loading procedure.

All Hayabusa2 samples were run in modified "single shot" mode (Schrader et al., 2014). Sample heating in the presence of $\mathrm{BrF}_{5}$ was carried out using a Photon Machines Inc. $50 \mathrm{~W}$ infrared $\mathrm{CO}_{2}$ laser $(10.6 \mu \mathrm{m})$ mounted on an $X-Y-Z$ gantry. Reaction progress was monitored by means of an integrated video system. After fluorination, the released $\mathrm{O}_{2}$ was purified by passing it through two cryogenic nitrogen traps and over a bed of heated $\mathrm{KBr}$ to remove any excess fluorine. The isotopic composition of the purified oxygen gas was analyzed using a Thermo Fisher MAT 253 dual inlet mass spectrometer with a mass resolving power of approximately 200 .

In most cases the amount of $\mathrm{O}_{2}$ gas liberated during sample reaction was less than $140 \mu \mathrm{g}$, the approximate limit for using the bellows facility on the MAT 253 mass spectrometer. In these cases, analysis was undertaken using the microvolume. Following analysis of the Hayabusa2 particle, the internal obsidian standard was fluorinated, and its oxygen isotope composition determined.

The $\mathrm{NF}^{+}$fragment ion of $\mathrm{NF}_{3}{ }^{+}$can cause interference with the mass 33 beam $\left({ }^{16} \mathrm{O}^{17} \mathrm{O}\right)$. In order to eliminate this potential problem all samples were treated using a cryogenic separation procedure. This was either done in the forward sense prior to analysis on the MAT 253 or as a second analysis with the already analyzed gas pulled back onto a dedicated molecular sieve and then rerun after cryogenic separation. Cryogenic separation involved taking the gas onto the molecular sieve at liquid nitrogen temperature and then releasing it to the main molecular sieve at a temperature of $-130^{\circ} \mathrm{C}$. Extensive tests have shown that $\mathrm{NF}^{+}$is retained on the first molecular sieve and that no significant fractionation results from the use of this technique.

Overall system precision in bellows mode, as defined by replicate analyses of our internal obsidian standard, is: $\pm 0.053 \%$ or for $\delta^{17} \mathrm{O} ; \pm 0.095 \%$ for $\delta^{18} \mathrm{O} ; \pm 0.018 \%$ o for $\Delta^{17} \mathrm{O}(2 \sigma)$ (Starkey et al., 2016). Oxygen isotopic analyses are reported in standard $\delta$ notation, where $\delta^{18} 0$ has been calculated as: 
$\delta^{18} O=\left[\left({ }^{18} \mathrm{O} /{ }^{16} O\right)_{\text {sample }} /\left({ }^{18} \mathrm{O} /{ }^{16} O\right)_{\text {VSMOW }}-1\right] 1000(\%)$

and similarly for $\delta^{17} \mathrm{O}$ using the ${ }^{17} \mathrm{O} /{ }^{16} \mathrm{O}$ ratio. VSMOW is the international standard Vienna Standard Mean Ocean Water. $\Delta^{17} 0$, which represents the deviation from the terrestrial fractionation line, has been calculated as: $\Delta^{17} \mathrm{O}=\delta^{17} \mathrm{O}-0.52 \delta^{18} \mathrm{O}$

\section{Sample preparation using a focused ion beam (FIB)}

Approximately 150 to 200 nm-thick sections were extracted from Ryugu particles using a Hitachi High Tech SMI4050 focused ion beam (FIB) instrument at the Kochi Institute for Core Sample Research (Kochi), JAMSTEC. After deposition of tungsten protection layers, regions-of-interest (up to $25 \times 25 \mu \mathrm{m}^{2}$ ) were cut out and thinned using a Ga${ }^{+}$ion beam at an accelerating voltage of $30 \mathrm{kV}$ and then finalized at 5 $\mathrm{kV}$ and probe current of $40 \mathrm{pA}$ to minimize surface damage layers. Subsequently, the ultrathin sections were mounted on scaled-up Cu grids (Kochi grid, Ito et al., 2020) using a micromanipulator equipped with the FIB.

\section{Elemental abundance by Instrumental Neutron Activation Analysis (INAA)}

Ryugu particles A0098 (1.6303 mg) and C0068 (0.6483 mg) were doubly sealed in cleaned and high purity polyethylene sheets in a pure $\mathrm{N}_{2}$-filled glove box at SPring-8 without any interaction with terrestrial atmosphere. Sample preparation of JB-1 (a geological standard rock sample issued by the Geological Survey of Japan) was carried out at Tokyo Metropolitan University.

INAA was done at Institute for Integrated Radiation and Nuclear Science, Kyoto University. Samples were irradiated two times with different irradiation periods in consideration of half-lives of the nuclides used for elemental quantification. First, samples were irradiated for $30 \mathrm{~s}$ at the pn-3 with thermal and first neutron fluxes of $4.6 \times 10^{12}$ and $9.6 \times 10^{11} \mathrm{~cm}^{-2} \mathrm{~s}^{-1}$, respectively, for the determination of $\mathrm{Mg}, \mathrm{Al}, \mathrm{Ca}, \mathrm{Ti}, \mathrm{V}$ and Mn. Chemical reagents such as MgO (99.99\% purity; Soekawa Chemical, Tokyo, japan), Al (99.9\% purity; Soekawa Chemical) and Si metals (99.999\% purity; FUJIFILM Wako Pure Chemical, Osaka, Japan) were also irradiated to correct for interfering nuclear reactions such as $(n, p)$. Sodium chloride $(99.99 \%$ purity; MANAC, Tokyo, Japan) was also irradiated with the samples to correct for neutron flux variations.

After neutron irradiation, the outer polyethylene sheet was replaced with a new sheet and gamma rays emitted from the samples and reference standards were immediately measured using Ge detectors. The same samples were reirradiated for 4 hours at a pn-2 with thermal and first neutron fluxes of $5.6 \times 10^{12}$ and $1.2 \times 10^{12} \mathrm{~cm}^{-2} \mathrm{~s}^{-1}$, respectively, for the determination of $\mathrm{Na}, \mathrm{K}, \mathrm{Ca}, \mathrm{Sc}, \mathrm{Cr}, \mathrm{Fe}, \mathrm{Co}, \mathrm{Ni}, \mathrm{Zn}, \mathrm{Ga}, \mathrm{As}, \mathrm{Se}, \mathrm{Sb}$, $\mathrm{Os}$, Ir and $\mathrm{Au}$. Reference samples for $\mathrm{Ga}, \mathrm{As}, \mathrm{Se}, \mathrm{Sb}, \mathrm{Os}$, Ir and Au were prepared by dropping a proper amount of concentration-known standard solutions of these elements onto the two sheets of filter papers 
which were then irradiated with the samples. Gamma-ray counting was carried out at Institute for Integrated Radiation and Nuclear Science, Kyoto University and RI Research Center, Tokyo Metropolitan University. For elemental quantification, a reference value of Cr for JB-1 was taken from Kong and Ebihara (1997), while literature values of Jochum et al. (2016) were used for the remaining elements. The analytical procedure of INAA is the same as that described by Shirai et al. (2020).

\section{Bulk mineralogy by XRD}

X-ray diffractometer (Rigaku SmartLab) was used to collect diffraction patterns of Ryugu samples A0029 (1<mg), A0037 (<<1 mg) and C0087 (<1 mg) at the National Institute of Polar Research. All the samples were ground to be fine powders using a sapphire glass plate on a silicon non-reflection plate, and then homogeneously placed onto the silicon non-reflection plate. The measurement conditions are as follows: $\mathrm{Cu} \mathrm{Ka}$ X-ray was produced at $40 \mathrm{kV}$ tube voltage and $40 \mathrm{~mA}$ tube current, the length of length limiting slit was $10 \mathrm{~mm}$, the divergence angle was $(1 / 6)^{\circ}$, in-plane rotation speed was $20 \mathrm{rpm}$, two theta range (twofold Bragg angle) was $3-100^{\circ}$, and it took 28 hours for single analysis. BraggBrentano optics was used. The detector was one-dimensional SSD (D/teX Ultra 250). Cu $\mathrm{K}_{\mathrm{b}} \mathrm{X}$-ray was removed using a Ni filter. The peaks were identified from the comparison with the measured data using the available samples, synthesized Mg-saponite (JCSS-3501, Kunimine Industries CO., LTD.), serpentine (antigorite, Miyazu, Nichika \#14-4-12-1), and pyrrhotite (monoclinic 4C, Chihuahua, Mexico), and using the PDF data from ICDD, dolomite (PDF 01-071-1662) and magnetite (PDF 00-019-0629). The diffraction data of Ryugu was also compared with those of hydrously altered carbonaceous chondrites, Orgueil Cl, Y-791198 CM2.4, and Y $980115 \mathrm{CY}$ (heating stage III). The comparison showed the similarity with Orgueil but not with Y791198 and $Y-980115$.

\section{Carbon functional groups by STXM-NEXAFS}

The carbon K-edge NEXAFS spectra of ultra-thin section samples made by FIB were measured using the STXM beam line, BL4U, at the UVSOR Synchrotron Facility, Institute for Molecular Science (Okazaki, Japan). The beam spot size focused with Fresnel zone-plate optics was about $50 \mathrm{~nm}$. The energy step size was $0.1 \mathrm{eV}$ in the fine-structure portions of the near-edge region (283.6-292.0 eV), and $0.5 \mathrm{eV}$ in the pre-edge and post-edge regions (280.0-283.5 and 292.5-300.0 eV). The acquisition time per image pixel for each energy step was set to be $2 \mathrm{~ms}$. Helium gas of $\sim 20$ mbar was backfilled with the STXM analysis chamber after evacuation. This helps to minimize thermal drift of X-ray optics-related apparatus in the chamber and the sample holder, and to reduce sample damage and/or oxidation (Wang et al., 2009). The carbon K-edge NEXAFS spectra were obtained from stack data using the aXis2000 software and the inhouse customized software for STXM data reduction. Note that an in-house sample transfer vessel and a glove box were used to avoid oxidization and contamination on the sample. 


\section{Hydrogen, carbon, and nitrogen isotopic imaging analysis using NanoSIMS}

The hydrogen, carbon, and nitrogen isotopic compositions of the Ryugu FIB sections were analyzed using isotopic imaging with the JAMSTEC NanoSIMS $50 \mathrm{~L}$ after STXM-NEXAFS analysis. A focused primary $\mathrm{Cs}^{+}$ beam of approximately $\sim 2 \mathrm{pA}$ was used for carbon, and nitrogen isotopic analyses, and approximately $\sim 13 \mathrm{pA}$ was used for hydrogen isotopic analyses, rastered over approximately $24 \times 24$ to $30 \times 30 \mu \mathrm{m}^{2}$ areas on the samples. Each analysis was initiated after stabilization of the secondary-ion beam intensity following three minutes of pre-sputtering with a relatively strong primary-ion beam current. For carbon and nitrogen isotopic analysis, images of ${ }^{12} \mathrm{C}^{-},{ }^{13} \mathrm{C}^{-},{ }^{16} \mathrm{O}^{-},{ }^{12} \mathrm{C}^{14} \mathrm{~N}^{-}$and ${ }^{12} \mathrm{C}^{15} \mathrm{~N}^{-}$were acquired simultaneously in multidetection with seven electron multipliers (EMs) at a mass resolving power of approximately 9000, sufficient to separate all relevant isobaric interferences (that is, ${ }^{12} \mathrm{C}^{1} \mathrm{H}$ on ${ }^{13} \mathrm{C}$ and ${ }^{13} \mathrm{C}^{14} \mathrm{~N}$ on ${ }^{12} \mathrm{C}^{15} \mathrm{~N}$ ). For hydrogen isotopic analysis, images of ${ }^{1} \mathrm{H}^{-},{ }^{2} \mathrm{D}^{-}$, and ${ }^{12} \mathrm{C}^{-}$were acquired using three EMs in multidetection at amass resolving power of approximately 3000 . Each analysis consisted of 30 scanned images of the same area, with individual images consisting of $256 \times 256$ pixels for the carbon and nitrogen isotopic analyses and $128 \times 128$ pixels for the hydrogen isotopic analysis. The dwell times were 3,000 $\mu \mathrm{s} /$ pixel for the carbon and nitrogen isotopic analyses and $5000 \mu \mathrm{s} /$ pixel for the hydrogen isotopic analysis. We used 1-hydroxybenzotriazole hydrate as the hydrogen, carbon, and nitrogen isotopic standards to correct for instrumental mass fractionations (Ito et al., 2014).

For silicon isotopic compositions of the presolar graphite in the C0068-25 FIB section, we acquired secondary ion images of ${ }^{12} \mathrm{C}^{-},{ }^{13} \mathrm{C}^{-},{ }^{16} \mathrm{O}^{-},{ }^{28} \mathrm{Si}^{-},{ }^{29} \mathrm{Si}^{-}$and ${ }^{30} \mathrm{Si}^{-}$simultaneously in multidetection with six electron EMs at a mass resolving power of approximately 9000 . These images consist of $256 \times 256$ pixels with a dwell time of $3,000 \mu \mathrm{s} /$ pixel. We used a silicon wafer as the hydrogen, carbon, and silicon isotopic standard to correct for instrumental mass fractionation.

The isotopic images were processed using the custom written software 'NASA JSC imaging software for NanoSIMS' (Ito and Messenger, 2008). Data were corrected for EM dead time (44 ns), and the QSA effect (Slodzian et al., 2004). Different scans of each image were aligned to correct image drift during acquisition. Final isotopic images were generated by adding the secondary ions of each image from each pixel over the scans.

\section{Micron to submicron scale mineralogical observations by TEM}

After STXM-NEXAFS and NanoSIMS analysis, the same FIB sections were studied using a transmission electron microscope (JEOL JEM-ARM200F) operated at an accelerating voltage of $200 \mathrm{kV}$ at Kochi, JAMSTEC. Microtextural observations were performed by bright-field TEM (BF-TEM) and high-angle annular dark-field scanning transmission electron microscopy (HAADF-STEM) imaging. Mineral phases were identified using selected-area electron diffraction and lattice-fringe imaging, and chemical analyses 
using energy-dispersive X-ray spectrometry (EDS) with a $100 \mathrm{~mm}^{2}$ silicon drift detector and JEOL Analysis Station 4.30 software. For quantitative analyses, the intensities of the characteristic X-rays of each element were measured using a fixed acquisition time of $30 \mathrm{~s}$, beam scan area of $\sim 100 \times 100 \mathrm{~nm}^{2}$, and beam current of $50 \mathrm{pA}$ in scanning TEM mode. The ( $\mathrm{Si}+\mathrm{Al})-\mathrm{Mg}$-Fe ratios of phyllosilicates were determined using experimental thickness-corrected $k$-factors obtained from a natural pyrope-almandine garnet standard.

\section{References in Method}

Ito, M. \& Messenger, S. Isotopic imaging of refractory inclusions in meteorites with the NanoSIMS 50L. Appl. Surf. Sci. 255, 1446-1450 (2008).

Ito, M., Tomioka, N., Uesugi, K., Uesugi, M., Kodama, Y., Sakurai, I., Okada, I., Ohigashi, T., Yuzawa, H., Yamaguchi, A., Imae, N., Karouji, Y., Shirai, N., Yada, T. \& Abe, M. The universal sample holders of microanalytical instruments of FIB, TEM, NanoSIMS, and STXM-NEXAFS for the coordinated analysis of extraterrestrial materials. Earth Planets Space 72:133 (2020).

Ito, M., Uesugi, M., Naraoka, H., Yabuta, H., Kitajima, F., Takano, Y., Mita, H., Karouji, Y., Yada T., Ishibashi, Y., Okada, T., \& Abe, M. H, C, and N isotopic compositions of Hayabusa category 3 organic samples. Earth Planets Space 66: 91 (2014).

Jochum, K. P., Weis, U., Schwager, B., Stoll, B., Wilson, S. A., Haug, G. H., Andreae, M. O., \& Enzweiler, J. Reference Values Following ISO Guidelines for Frequently Requested Rock Reference Materials. Geostand. Geoanalytical Res. 40, 333-350 (2016).

King, A. J., Schofield, P. F., Howard, K. T. \& Russell, S. S. Modal mineralogy of Cl and Cl-like chondrites by X-ray diffraction. Geochim. Cosmochim. Acta 165, 148-160 (2015).

Kong, P. \& Ebihara, M. Reproducibility of elemental concentrations for JB-1, a GSJ rock reference sample, with special reference to Mo, W and Ta. Geochem. J. 31, 339-344 (1997).

Miller, M. F. Isotopic fractionation and the quantification of 170 anomalies in the oxygen three-isotope system: an appraisal and geochemical significance. Geochim. Cosmochim. Acta 66, 1881-1889 (2002).

Miller, M. F., Franchi, I. A., Sexton, A. S. \& Pillinger, C. T. High precision $\Delta 170$ isotope measurements of oxygen from silicates and other oxides: Methods and applications. Rapid Comm. Mass Spec. 13, 12111217 (1999).

Schrader, D. L., Davidson, J, Greenwood, R. C., Franchi, I. A. \& Gibson, J. M. A water-ice rich minor body from the early Solar System: The CR chondrite parent asteroid. Earth Planet. Sci. Lett. 407, 48-60 (2014). 
Shirai, N., Karouji, Y., Kumagai, K., Uesugi, M., Hirahara, K., Ito, M., Tomioka, N., Uesugi, K., Yamaguchi, A., Imae, N., Ohigashi, T., Yada, T. \& Abe, M. The effects of possible contamination by sample holders on samples to be returned by Hayabusa2. Meteorit. Planet. Sci. 55, 1665-1680 (2020).

Starkey, N. A., Jackson, C. R. M., Greenwood, R. C., Parman, S., Franchi, I. A., Jackson, M., Fitton, J. G., Stuart, F. M., Kurz, M. \& Larsen, L. M. Triple oxygen isotopic composition of the high $3 \mathrm{He} / 4 \mathrm{He}$ mantle. Geochim. Cosmochim. Acta 176, 227-238 (2016).

Slodzian, G., Hillion, F., Stadermann, F. J. \& Zinner, E. QSA influences on isotopic ratio measurements. Appl. Surf. Sci. 231-232, 874-877 (2004).

Uesugi, M., Hirahara, K., Uesugi, K, Takeuchi, A., Karouji, Y., Shirai, N., Ito, M., Tomioka, N., Ohigashi, T., Yamaguchi, A., Imae, N., Yada, T. \& Abe, M. Development of a sample holder for synchrotron radiationbased computed tomography and diffraction analysis of extraterrestrial materials. Rev. Sci. Instrum. 91. doi: 10.1063/1.5122672 (2020).

Uesugi, M., Uesugi, K. \& Oka, M. Non-destructive observation of meteorite chips using quantitative analysis of optimized X-ray micro-Computed Tomography. Earth Planet. Sci. Lett. 299, 359-367 (2010).

Uesugi, M., Uesugi, K., Takeuchi, A., Suzuki, Y., Hoshino, M. \& Tsuchiyama, A. Three-dimensional observation of carbonaceous chondrites by Synchrotron radiation X-ray CT - quantitative analysis and developments for the future sample return missions. Geochim. Cosmochim. Acta 116, 17-32 (2013).

Wang, J., Morin, C., Li, L., Hitchcock, A. P., Scholl, A. \& Doran, A. Radiation damage in soft X-ray microscopy. J. Electron Spectros. Relat. Phenomena 170, 25-36 (2009).

\section{Declarations}

\section{Data availability}

Correspondence and requests for materials should be addressed to M.I. All analytical data related to this manuscript will be put on the JAXA Data ARchives and Transmission System (DARTS) after a one-year proprietary period.

\section{Acknowledgements}

We thank all scientists and engineers of the Hayabusa2 project whose dedication and skill brought these precious particles back to Earth. The meteorite samples of Orgueil, Y-791198, Y-82162, and Y 980115 were provided by the National Institute of Polar Research, Japan. The synchrotron radiation CT experiments were performed at the BL20XU of SPring-8 with the approval of the Japan Synchrotron Radiation Research Institute (JASRI) (2021A0166, 2021B0188). SR experiments at SPring-8 are supported by RIKEN. The synchrotron radiation STXM-NEXAFS experiment at BL4U of UVSOR were 
carried out under the MOU between JAMSTEC Kochi Institute for Core Sample Research and Institute for Molecular Science UVSOR synchrotron facility. We thank Marine Works Japan for assistance of curative activity, initial none-destructive investigations, and a sample preparation of Ryugu particles. We thank that the UK embassy in Tokyo provided a support for the Ryugu particle transportation from Japan to the UK. A part of this work was performed by using facilities of the Institute for Integrated Radiation and Nuclear Science, Kyoto University. This research was supported in part by the JSPS KAKENHI (JP18K18795 and JP18H04468 to MI, JP20H01965 to NT, JP18H05479 [Innovative Areas "MFS Materials Science"] to MU, JP19H01959 to AY, JP21K03652 to NI, JP17H06459 to TU, JP19K03958 to MA, JP17H06459 to TO, JP18K03830 to TY, JP19K23473, JP20K14548 to TH, JP19K23474, JP21K13986 to DY, JP20K14535 to RF, JP17H06459 and JP19H01951 to SW), and by the NIPR Research Project (KP307 to AY).

\section{Author contributions}

M.I. leads the project and wrote a paper with input from all coauthors. M.I., N.T., M.U., A.Y., N.I., N.S., MC.L., R.C.G., Y.K., A.N., K.M., N.M., and K.Y. conducted sample handling, preparation, and mounting processes of Ryugu grains. M.I., N.T., M.U., O.T., K.U., K.H., I.S., and I.O. developed universal sample holders for multiple instruments. M.U., M.Y., A. Tsuchiyama, A. Takeuchi and U.K. arranged and performed SR-CT analysis at SPring-8. SEM-EDS analysis was conducted by A.Y., M.K., N.I., and M.I. EPMA analysis and data reduction was conducted by A.Y. XRD analysis was conducted by N.I. FIB sample processing was conducted by Y.K. and N.T. STXM-NEXAFS analysis conducted by T.O., H.Y., M.I., M.U and A.N. NanoSIMS analysis conducted by M.I. TEM work was done by N.T. Oxygen isotopes were measured by R.C.G., R.F., I.A.F., and J.A.M at the Open University. N.S. and S.S. conducted INAA analysis at Kyoto University. S. Tanaka, T.O., S.Sugita, N.N., K.Kitazato, H. Y.I., Naru Hirata, Naoyuki Hirata, K.M., T. Michikami, H.Noda, R.N., Y.S., K. Shirai, K.O., K.W., H. Senshu, Y. Yamamoto, T. Morota, R.H., Y. Yokota, C. Honda, M.M., N.S., E.T., A. Miura, M. Yamada, M.A., T.I., H. Yano, A.F., C. Hirose, S.H., H.I., S.K., Y.M., O.M., M.O., N.O., G.O., H. Sawada, T. Shimada, T.T., Y. Takei, H.T., R.T., K. Yoshikawa, F.T., T. Saiki, S.N., M.Y., S.W. and Y. Tsuda contributed to the spacecraft operations and selected sampling sites. T.Y., A.N., K.Y., M.N., A.N., A.M., K.K, K.H., Y.H., M.S., M. Yoshitake, T.O., M.A., and T.U lead the JAXA curation activities for initial characterization of allocated Ryugu particles. M. Yoshikawa, T. Saiki, S. Tanaka, F.T., S.N., S. Sugita, N.N., K. Kitazato, T.O., Naru Hirata, S.W., and Y.T. administered the project and acted as principal investigators. All authors contributed to the data interpretation, commented, and finalized to the paper.

\section{Competing interests}

The authors declare no competing interests. 
Supplementary information is available for this paper: Supplementary Figures S1 - S3, and Supplementary Tables S1 - S3.

\section{Figures}

Fig. 1. The Ryugu $\mathbf{C 0 0 6 8}$ thin section images. (A) a combined X-ray map composed of $\mathrm{Mg} \mathrm{Ka}$ (red), Ca Ka (green), and Fe Ka (blue) of a dry-polished section of the Ryugu C0068 particle. The section consists of phyllosilicate (red: 88 vol\%), carbonate (dolomite; light green: $~ 1.6$ vol\%), magnetite (blue: $~ 5.3$ vol\%), and sulfide (blue: sulfide= $\sim 2.5$ vol\%). (B) backscattered electron image of regions of outlined in (A). Bru: brunnerite, Dol: dolomite, Mag: magnetite, Sap: saponite, Srp: serpentine. (C) High-resolution TEM image of typical saponite-serpentine intergrowth area showing $0.7 \mathrm{~nm}$ and $1.1 \mathrm{~nm}$ lattice fringes of serpentine and saponite, respectively.

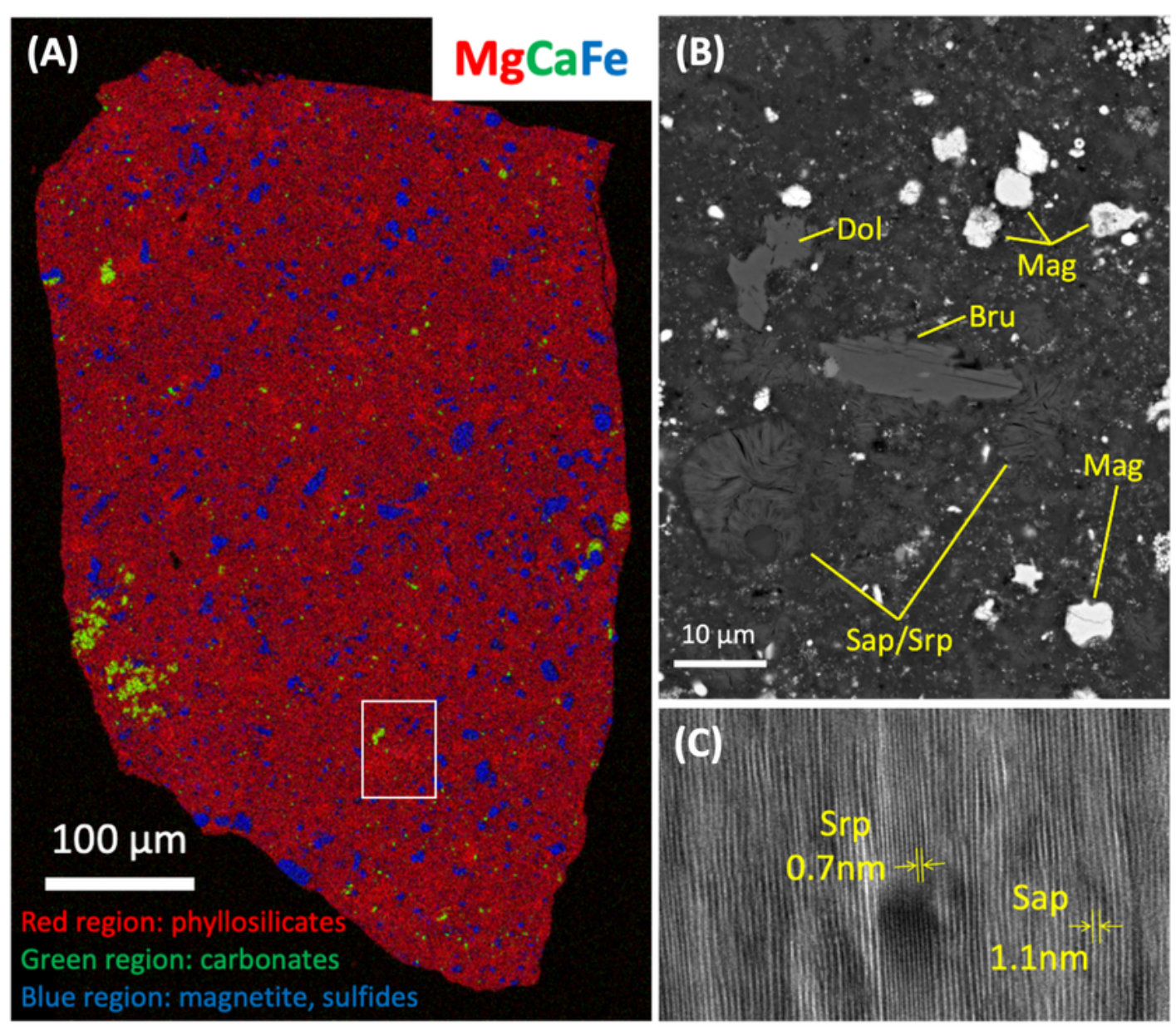


Figure 1

See figure for legend.

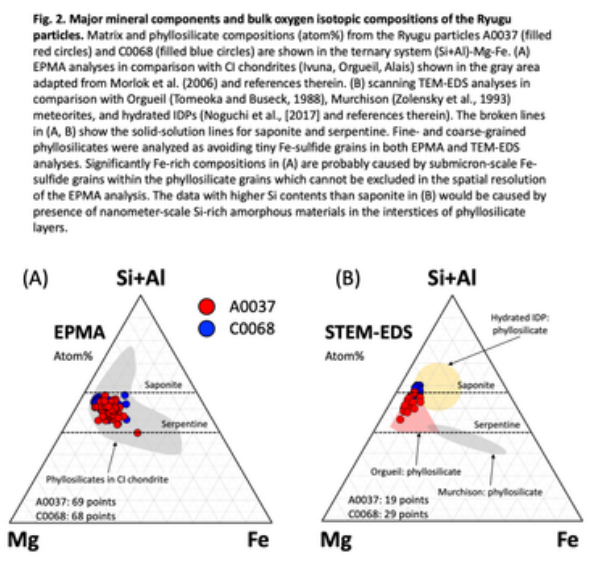

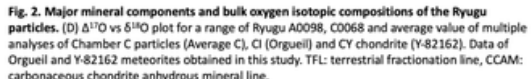
this study. TFL'terrestrial fractionation line,

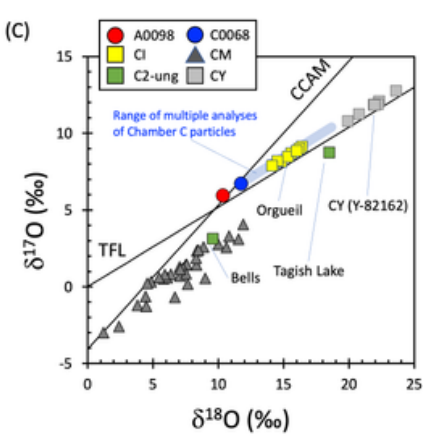

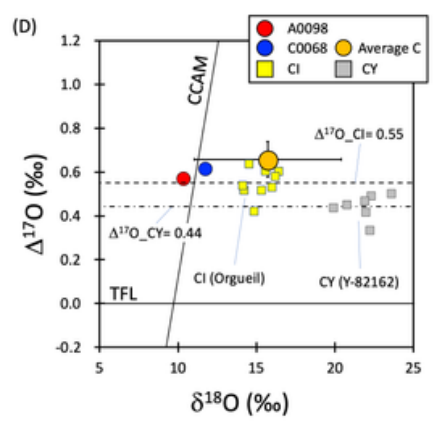

Figure 2

See figure for legend. 
Fig. 3. Scanning transmission X-ray microscopy (STXM) and NanoSIMS ion imaging analyses of a focused ion beam (FIB) section of the Ryugu C0068-25. (A) STXM carbon K-edge spectral image indicates the section is dominated by carbon. (B) Carbon-NEXAFS spectra normalized at $292 \mathrm{eV}$ of aromatic $(\mathrm{C}=\mathrm{C})$-rich regions (red), aliphatic-rich regions (green), and matrix (blue). The Grey line is spectrum of Murchison IOM (Cody et al., 2008) in comparison. (C) Combined RGB map in R: Aromatic rich $(\mathrm{C}=\mathrm{C})$ area, $\mathrm{G}$ : Aliphatic-rich area, B: Matrix. (D) Aliphatic-rich organics concentrated in coarsegrained phyllosilicates in area enlarged from the white box in (A,C). (E) Large nanoglobule (n.g-1) in area enlarged from the white box in (A,C).

(A) STXM carbon K-edge spectral image

(B) Carbon-NEXAFS spectra

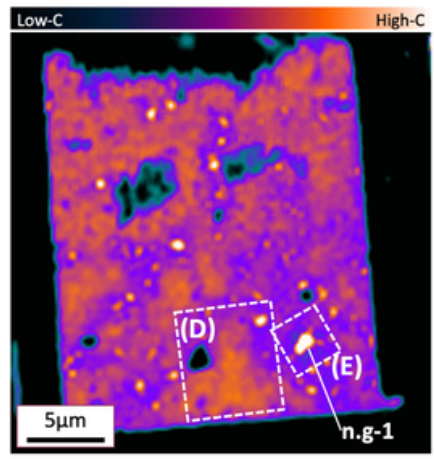

(C) Combined RGB map in R: Aromaticrich $(\mathrm{C}=\mathrm{C})$ area, $\mathrm{G}$ : Aliphatic-rich area, $\mathrm{B}$ : Matrix
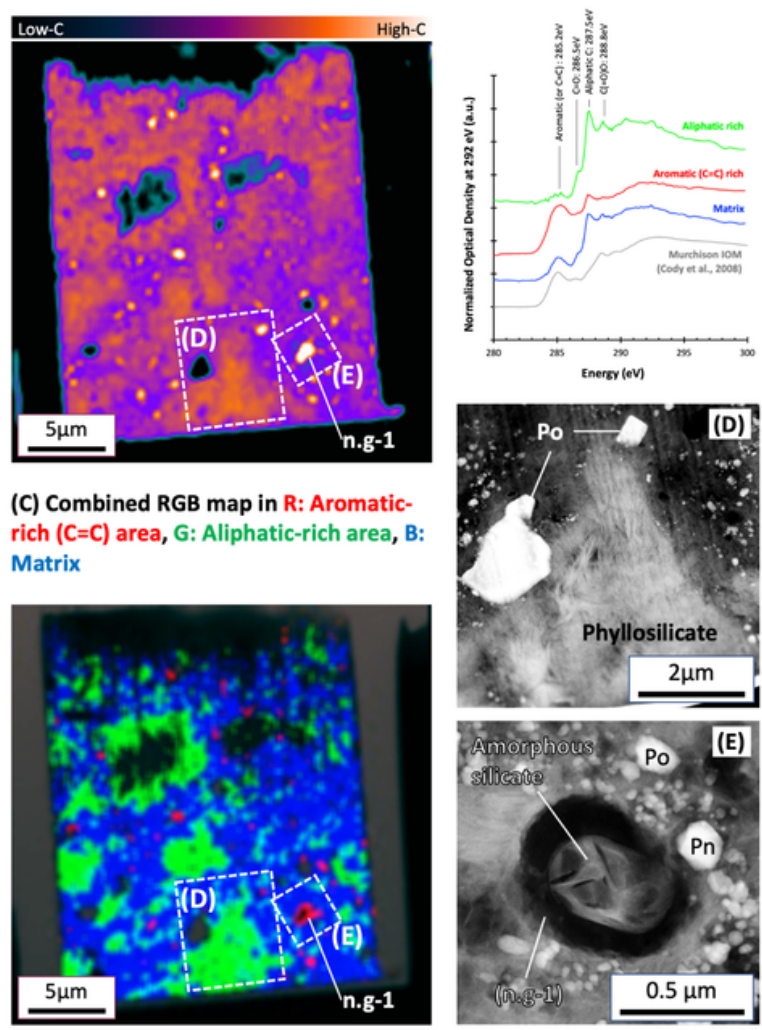

Fig. 3. Scanning transmission X-ray microscopy (STXM) and NanoSIMS ion imaging analyses of a focused ion beam (FIB) section of the Ryugu C0068-25. (F) Elemental images of hydrogen, carbon and nitrogen, elemental ratio image of ${ }^{12} \mathrm{C} /{ }^{1} \mathrm{H}$ and isotope images of $\delta^{13} \mathrm{C}$ and $\delta^{15} \mathrm{~N}$ of the section. P.G-1: presolar graphite with extreme ${ }^{13} \mathrm{C}$ enrichment. n.g-1: nanoglobule of the white box in (D).

(F)

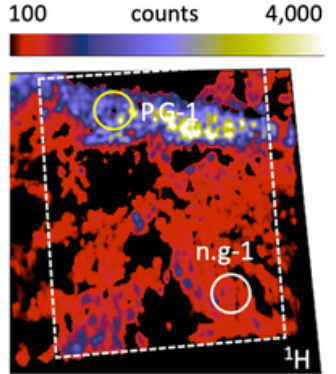

0.82 ratio $\quad 16.65$

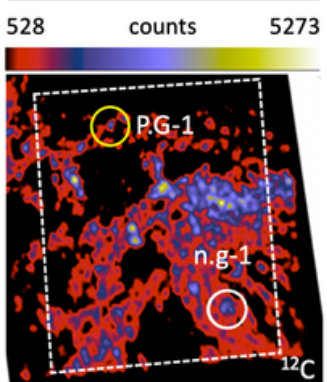

$-1000 \quad \% \quad+30,000$

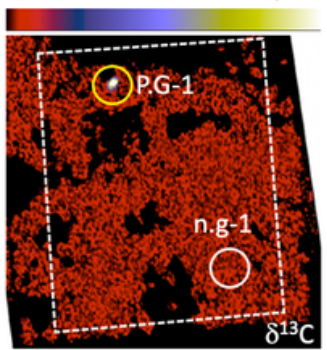

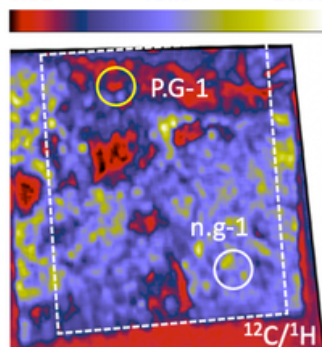
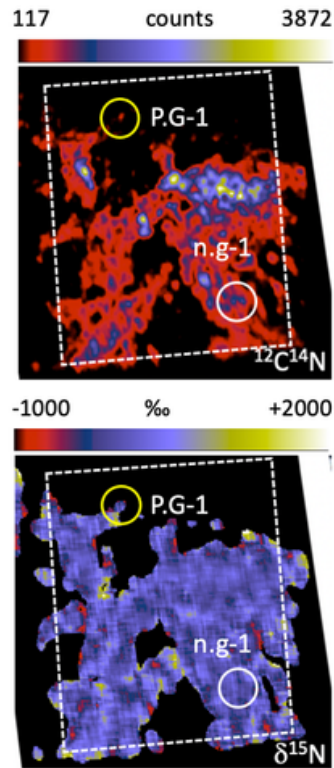

\section{Figure 3}

See figure for legend. 
Fig. 4. Light element isotopic compositions of the Ryugu particles A0002, A0037 and $C 0068$. Comparison of the bulk $\mathrm{H}$ and $\mathrm{N}$ isotopic compositions of the Ryugu particles (red circles: A0002, A0037; blue circles: C0068) with those of the IDPs, Wild2 cometary samples, and carbonaceous chondrites ( $\mathrm{Cl}, \mathrm{CM}, \mathrm{CR}, \mathrm{C2}$-ung). The isotopic compositions are given in Table S3, and IDPs from Floss et al. (2006), Wild2 cometary samples from McKeegan et al. (2006) and carbonaceous chondrites from Alexander et al. (2017 and reference there in). Dotted lines are terrestrial isotopic values of $\mathrm{H}$ and $\mathrm{N}$.

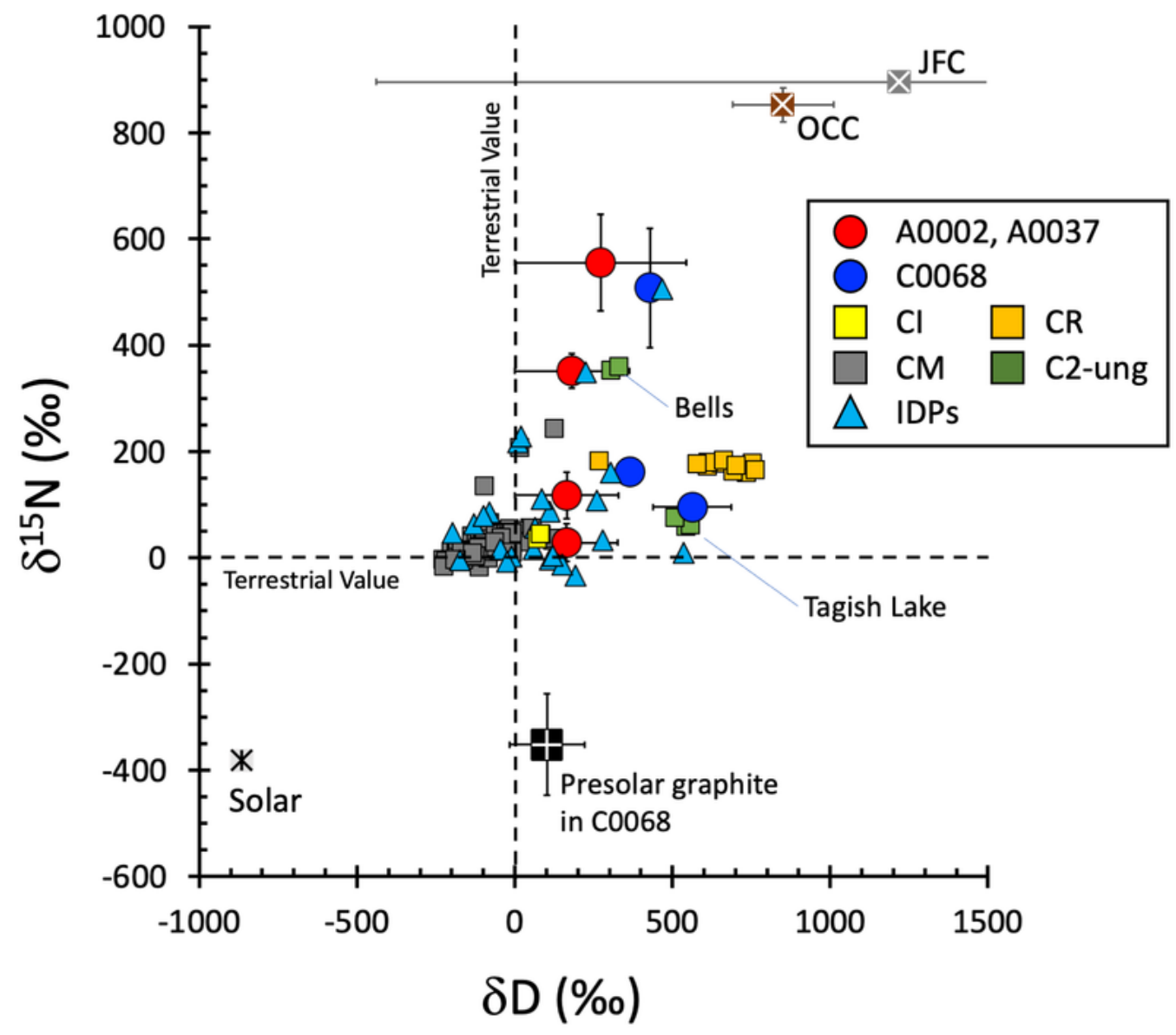

Figure 4

See figure for legend.

\section{Supplementary Files}

This is a list of supplementary files associated with this preprint. Click to download. 
- ExDFig1.png

- FigS1AB.png

- ExDFig2.png

- FigS2.png

- FigS3.png

- SupplementalTables.docx 